\title{
Estimation in Misclassified Size-Biased Generalized Negative Binomial Distribution
}

\author{
B. S. Trivedi ${ }^{1, *}$, M. N. Patel $^{2}$ \\ ${ }^{1}$ H. L. Institute of Commerce, Ahmedabad University, Navrangpura, Ahmedabad \\ ${ }^{2}$ Department of Statistics, School of Sciences, Gujarat University, Ahmedabad \\ Corresponding Author: bhaktida.trivedi@ahduni.edu.in
}

Copyright (C) 2013 Horizon Research Publishing All rights reserved.

\begin{abstract}
In this paper, we are concerned with the situations, where sometimes value two is reported erroneously as one in relation to size biased generalized negative binomial distribution (SBGNBD) with probability $\alpha$. We have obtained the Maximum likelihood estimator and Bayes estimator under general entropy loss function. A simulated study is carried out to access the performance of the maximum likelihood estimators and Bayes estimators. Also comparison has been made between maximum likelihood estimator and Bayes estimator.
\end{abstract}

Keywords Misclassification, maximum likelihood estimator, Bayes estimator, general entropy loss function, simulation

\section{Introduction}

Pascal defined the negative binomial distribution as

$$
\begin{array}{r}
P[X=x]=\left(\begin{array}{c}
m+x-1 \\
x
\end{array}\right) \theta^{x}(1-\theta)^{m} \\
x=0,1,2, \ldots \ldots \text { Where } 0<\theta<1, m>0
\end{array}
$$

The mean and variance of the distribution are given as

$$
E(X)=\mu_{1}^{\prime}=\frac{m \theta}{1-\theta} \quad \text { and } \quad V(X)=\mu_{2}=\frac{m \theta}{(1-\theta)^{2}}
$$

It can be easily seen that variance is always greater than mean.

This type of model was used to represent 'accident-proneness' by Greenwood and Yule (1920). Wise (1946) describes an application of this kind of model to represent the distribution of number of pinholes per 'unit' length of enameled wire. The negative binomial distribution is very often a first choice as alternative when it is felt that a Poisson distribution might be inadequate.

A generalization of the negative binomial distribution so called generalized negative binomial distribution (GNBD) defined by Jain \& Consul (1971) is given by

$$
\begin{gathered}
P[X=x]=p(x ; \alpha, \theta)=\frac{m}{m+\beta x}\left(\begin{array}{c}
m+\beta x \\
x
\end{array}\right) \theta^{x}(1-\theta)^{m+\beta x-y x} \\
=\frac{m \Gamma(m+\beta x) \theta^{x}(1-\theta)^{m+\beta x-x}}{x ! \Gamma(m+\beta x-x+1)}
\end{gathered}
$$

with $0<\theta<1,0 \leq \alpha \leq 1, m>0 \&|\beta \theta|<1, x \in T=\{0,1,2,3, \ldots \ldots \ldots\}$.

When the sampling mechanism selects units with probability proportional to some measure of the unit size, the resulting distribution is called size - biased. Such distributions arise in the life length and were studied by various authors. (see Gupta (1979, 1984), Gupta \&. Tripathi (1987,1992), Williford and Binghan(1979)).

A size - biased GNBD is obtained by taking the weight of (1.3) as $x$, given by

$$
P[X=x]=\frac{x \cdot P(x)}{E(x)}
$$

Using (1.1) and (1.2) the resulting size-biased GNBD, can be defined as 


$$
\begin{aligned}
P(X=x)=P[x ; \alpha, \theta]=\frac{m \Gamma(m+\beta x) \theta^{x}(1-\theta)^{m+\beta x-x}}{x ! \Gamma(m+\beta x-x+1)} \times \frac{x}{\frac{m \theta}{1-\theta \beta}} & =\frac{\Gamma(m+\beta x) \theta^{x-1}(1-\theta)^{m+\beta x-x}(1-\theta \beta)}{(x-1) ! \Gamma(m+\beta x-x+1)} \\
x \in T & =\{1,2,3, \ldots \ldots \ldots\}
\end{aligned}
$$

In experiments reported to discrete distributions, situations may often arise where the probabilities of different counts are affected in various ways. There may arise a situation where certain count is reported as some other count. e.g. when defective item is inspected wrongly as non defective item and vice versa. Cohen (1960) discussed this kind of situation where the count one is reported as count zero in case of Poisson and Binomially distributed random variables, whereas Jani and Shah (1979) studied the problem of misclassification in a class of discrete distribution, the power series distribution defined by Noack (1950) in which some of observations corresponding to the cell one are sometimes wrongly reported as observations corresponding to cell zero.

The problem of misclassification for the most general class of discrete distribution, misclassified generalized negative binomial distribution is considered by Gupta and Tripathi (1987) in some particular case of modified power series distribution and recently by Patel \& Patel $(1996,2001)$ in the case of generalized power series distribution and modified power series distribution for a more general situation where sometimes the value $(c+1)$ is reported as $c$.

If we assume that some of the observations corresponding to the cell $x=2$ are wrongly reported as belonging to the cell $x=1$, and let this probability of misclassification be $\alpha$. Then the probability function of the reported number of observations can be written as

$$
P[X=x]=\left\{\begin{array}{lr}
P(x=1)+\alpha P(x=2), & \text { for } x=1 \\
P(x=2)-\alpha P(x=2), & \text { for } x=2 \\
P(x), & \text { for } x=3,4,5, \ldots
\end{array}\right.
$$

Suppose $\mathrm{X}$ is a random variable having the size - biased generalized negative binomial distribution (1.4) from which a random sample is drawn. Then the resulting distribution of $\mathrm{X}$ based on (1.5) is called misclassified size-biased generalized negative binomial distribution (MSBGNBD), which can be written in the form

$$
P[X=x]=p(x ; \alpha, \theta)=\left\{\begin{array}{cc}
(1-\theta)^{m+\beta-1}(1-\theta \beta)\left(1+\alpha(m+2 \beta-1) \theta(1-\theta)^{\beta-1}\right. & , x=1 \\
(1-\alpha)(m+2 \beta-1)(1-\theta \beta) \theta(1-\theta)^{m+2(\beta-1)} & , x=2 \\
\frac{\Gamma(m+\beta x) \theta^{x-1}(1-\theta)^{m+\beta x-x}(1-\theta \beta)}{(x-1) ! \Gamma(m+\beta x-x+1)}, x=3,4, . . \infty & \text { i.e. } x \in s
\end{array}\right.
$$

where $s=T-\{1,2\}$

$$
0 \leq \alpha \leq 1, \quad 0<\theta<1, \quad m>0, \quad|\beta \theta|<1 .
$$

Under the different choice of the parameters of MSBGNBD, the graphs of the probability distribution are given below.
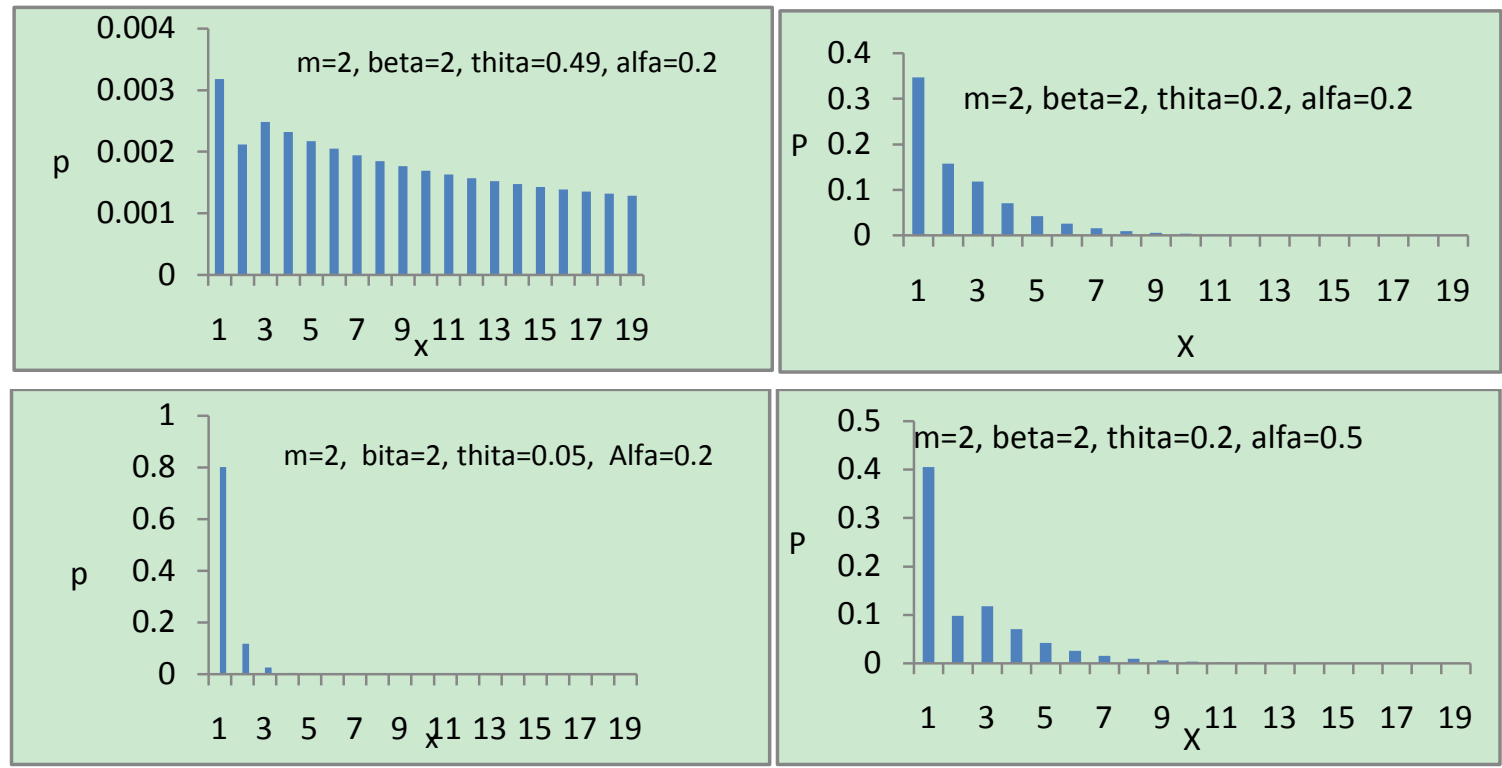

Figure 1. Plots of the p.m.f. of the misclassified size-biased generalized negative binomial distribution. 
This distribution is considered by Hassan and Ahmad (2009). Moments and some recurrence relations of the moments are derived.

In this paper we have considered the Maximum likelihood (ML) estimation and Bayes estimation of the parameters of MSBGNBD. In section 2 we have obtained ML estimators of the parameters and studied their properties by theorem. Section 3 describes Prior, Posterior and Marginal distribution of the parameters $\theta$ and $\alpha$. In section 4, we have derived Bayes estimators under General entropy loss function (GELF). Comparison among different estimation methods using simulation study is presented in section 6 .

\section{Maximum Likelihood Estimators}

Let $1,2,3, \ldots \ldots \ldots k$ (where $k \in\{1,2, \ldots \ldots . \infty\}$ ) be the possible values of a random variable $x$ in a random sample of size $\mathrm{N}$ taken from the distribution given in (1.6). Let $\alpha$ be the proportion of misreported number of observations corresponding to the cell $x=2$ by reporting them as belonging to the cell $x=1$.

Let $n_{k}$ denote the number of observations corresponding to the value $x_{k}$ in the sample, then we have

The likelihood function $L$ of such a sample is given by

$$
\sum_{i=1}^{k} n_{i}=N
$$

$$
L=\prod_{i=1}^{k} p_{i}^{n_{i}}
$$

where $p_{i}=p(x=i) \quad, i=1,2,3, \ldots \ldots \ldots . k$

$$
\begin{gathered}
L=\left[\frac{\theta(1-\theta)^{\beta-1}\left(m+\alpha m(m+2 \beta-1)\left(\theta(1-\theta)^{\beta-1}\right)\right)}{(1-\theta)^{-m} \cdot\left(\frac{m \theta}{1-\theta \beta}\right)}\right]^{n_{1}} \times \\
{\left[\frac{(1-\alpha) m(m+2 \beta-1)}{(1-\theta)^{-m} \cdot\left(\frac{m \theta}{1-\theta \beta}\right)}\left\{\theta(1-\theta)^{\beta-1}\right\}^{2}\right]^{n_{2}} \times} \\
\prod_{i=3}^{k}\left[\frac{m \Gamma(m+\beta i)}{(i-1) ! \Gamma(m+\beta i-i+1)} \frac{\left\{\theta(1-\theta)^{\beta-1}\right\}^{i}}{(1-\theta)^{-m} \cdot\left(\frac{m \theta}{1-\theta \beta}\right)}\right]^{n_{i}}
\end{gathered}
$$

Taking its natural logarithm and differentiating it, in terms with respect to $\alpha$ and $\theta$, assuming other parameters $\mathrm{m}$ and $\beta$ are known and comparing them with zero, we have the likelihood equations as

$$
\begin{aligned}
& \frac{\partial \ln L}{\partial \theta}=\left(n_{1}+2 n_{2}+\sum_{i=3}^{k} i n_{i}\right)\left(\frac{1}{\theta}-\frac{(\beta-1)}{(1-\theta)}\right)+ \\
& \frac{n_{1} \alpha m(m+2 \beta-1)\left[(1-\theta)^{\beta-1}-\theta(\beta-1)(1-\theta)^{\beta-2}\right]}{m+\alpha m(m+2 \beta-1) \theta(1-\theta)^{\beta-1}}- \\
& \quad \sum_{i=1}^{k} n_{i}\left[\frac{m}{(1-\theta)}+\frac{1}{\theta}+\frac{\beta}{1-\theta \beta}\right]=0 \\
& \frac{\partial \ln L}{\partial \alpha}=\frac{m\left(n_{1}(m+2 \beta-1) \theta(1-\theta)^{\beta-1}\right)}{m\left(1+\alpha(m+2 \beta-1) \theta(1-\theta)^{\beta-1}\right)}-\frac{n_{2}}{1-\alpha}=0
\end{aligned}
$$

by simplifying, we get

$$
\alpha=\frac{n_{1}(m+2 \beta-1) \theta(1-\theta)^{\beta-1}-n_{2}}{(m+2 \beta-1) \theta(1-\theta)^{\beta-1}\left(n_{1}+n_{2}\right)}
$$

By taking

$$
\text { where } N=\sum_{i=1}^{k} n_{i} \text {. }
$$

$$
A_{1}=\left(n_{1}+2 n_{2}+\sum_{i=3}^{k} i n_{i}\right) \text { and } A_{2}=1+\alpha(m+2 \beta-1) \theta(1-\theta)^{\beta-1}
$$


The likelihood equation (2.1) becomes,

$$
\begin{aligned}
& \frac{\partial \ln L}{\partial \theta}=\frac{n_{1}}{\theta}(m+2 \beta-1) \alpha\left(\frac{\theta(1-\theta)^{\beta-1}}{A_{2}}-\frac{\theta^{2}(\beta-1) \frac{(1-\theta)^{\beta-1}}{1-\theta}}{A_{2}}\right)+ \\
& A_{1}\left(\frac{1}{\theta}-\frac{\beta-1}{1-\theta}\right)+-N\left[\frac{m}{(1-\theta)}+\frac{1}{\theta}+\frac{\beta}{1-\theta \beta}\right]=0
\end{aligned}
$$

using equation (2.4) in (2.5) and after some algebraic manipulation we get,

$$
A_{1}\left(\frac{1}{\theta}-\frac{\beta-1}{1-\theta}\right)+\frac{n_{1} \alpha n_{2}}{\frac{n_{1}(1-\alpha)}{n_{1}-\alpha\left(n_{1}+n_{2}\right)}\left[n_{1}-\alpha\left(n_{1}+n_{2}\right)\right]}\left[\frac{1}{\theta}-\frac{\beta-1}{1-\theta}\right]-N\left[\frac{m}{(1-\theta)}+\frac{1}{\theta}+\frac{\beta}{1-\theta \beta}\right]=0
$$

The above equation can be written as

$$
\theta=\left(A_{1}+\frac{\alpha n_{2}}{(1-\alpha)}-N\right) \times\left(\frac{(\beta-1)}{(1-\theta)} A_{1}+\frac{\alpha n_{2}(\beta-1)}{(1-\alpha)(1-\theta)}+\frac{N m}{1-\theta}+\frac{N \beta}{1-\theta \beta}\right)^{-1}
$$

By solving the equations (2.3) and (2.5) simultaneously, we get ML estimates of the parameters $\alpha$ and $\theta$. Now, to obtain variance covariance matrix of the ML estimators we proceed as follows.

We differentiae the equations (2.2) and (2.4) again so we get

$$
\begin{aligned}
\frac{\delta^{2} \ln L}{\delta \theta^{2}}= & \left(n_{1}+2 n_{2}+\sum_{i=3}^{k} i n_{i}\right)\left(-\frac{1}{\theta^{2}}+\frac{(\beta-1)}{(1-\theta)^{2}}\right) \\
& +\frac{n_{1} \alpha}{2}\left[\begin{array}{c}
\left\{\left(1+\frac{\alpha}{2}(m+2 \beta-1) \theta(1-\theta)^{\beta-1}\right)(1-\beta)(1-\theta)^{\beta-2}(2-\theta(\beta-2))\right\} \\
-\left\{(1-\theta)^{2(\beta-2)}(1-\theta \beta)^{2} \frac{\alpha}{2}(m+2 \beta-1)\right\}
\end{array}\right] \\
& +\sum_{i=1}^{k} n_{i}\left[\frac{m}{(1-\theta)^{2}}+\frac{1}{\theta^{2}}+\frac{\beta^{2}}{(1-\theta \beta)^{2}}\right]
\end{aligned}
$$

and

$$
\begin{aligned}
& E\left(\frac{\delta^{2} \ln L}{\delta \theta^{2}}\right)=N\left(\sum_{i=1}^{k} i p_{i}\right)\left(-\frac{1}{\theta^{2}}+\frac{(\beta-1)}{(1-\theta)^{2}}\right)+ \\
& +\frac{N p_{1} \alpha}{2}\left[\begin{array}{c}
\left\{\left(1+\frac{\alpha}{2}(m+2 \beta-1) \theta(1-\theta)^{\beta-1}\right)(1-\beta)(1-\theta)^{\beta-2}(2-\theta(\beta-2))\right\} \\
-\left\{(1-\theta)^{2(\beta-2)}(1-\theta \beta)^{2} \frac{\alpha}{2}(m+2 \beta-1)\right\} \\
\left(1+\frac{\alpha}{2}(m+2 \beta-1) \theta(1-\theta)^{\beta-1}\right)^{2}
\end{array}\right] \\
& +\sum_{i=1}^{k} N p_{i}\left[\frac{m}{(1-\theta)^{2}}+\frac{1}{\theta^{2}}+\frac{\beta^{2}}{(1-\theta \beta)^{2}}\right]
\end{aligned}
$$

Similarly

$$
\frac{\delta^{2} \ln L}{\delta \alpha^{2}}=\frac{-n_{1}(m+2 \beta-1)^{2} \theta^{2}(1-\theta)^{2(\beta-1)}}{\left(1+\alpha(m+2 \beta-1) \theta(1-\theta)^{\beta-1}\right)^{2}}-\frac{n_{2}}{(1-\alpha)^{2}}
$$

and

$$
\frac{\delta^{2} \ln L}{\delta \theta \delta \alpha}=\frac{\left(\begin{array}{c}
E\left(\frac{\delta^{2} \ln L}{\delta \alpha^{2}}\right)=\frac{-N p_{1}(m+2 \beta-1)^{2} \theta^{2}(1-\theta)^{2(\beta-1)}}{\left(1+\alpha(m+2 \beta-1) \theta(1-\theta)^{\beta-1}\right)^{2}}-\frac{N p_{2}}{(1-\alpha)^{2}} \\
n_{1}(m+2 \beta-1)\left(1+\alpha(m+2 \beta-1) \theta(1-\theta)^{\beta-1}\right) \times \\
{\left[(1-\theta)^{\beta-1}+\theta(\beta-1)(1-\theta)^{\beta-2}\right]-\left[\alpha \theta(m+2 \beta-1)(1-\theta)^{2(\beta-1)}\right]+} \\
{\left[\theta^{2} \alpha(m+2 \beta-1)(\beta-1)(1-\theta)^{2 \beta-3}\right]}
\end{array}\right)}{\left(1+\alpha(m+2 \beta-1) \theta(1-\theta)^{\beta-1}\right)^{2}}
$$

and

$$
E\left(\frac{\delta^{2} \ln L}{\delta \theta \delta \alpha}\right)=\frac{\left(\begin{array}{c}
N p_{1}(m+2 \beta-1)\left(1+\alpha(m+2 \beta-1) \theta(1-\theta)^{\beta-1}\right) \times \\
{\left[(1-\theta)^{\beta-1}+\theta(\beta-1)(1-\theta)^{\beta-2}\right]-} \\
{\left[\alpha \theta(m+2 \beta-1)(1-\theta)^{2(\beta-1)}+\theta^{2} \alpha(m+2 \beta-1)(\beta-1)(1-\theta)^{2 \beta-3}\right]}
\end{array}\right)}{\left(1+\alpha(m+2 \beta-1) \theta(1-\theta)^{\beta-1}\right)^{2}}
$$


where $p_{i}=P(x=i)$ as given in equation (1.6).

Hence the variance covariance matrix of ML estimators is given by $\Sigma^{-1}$ where

$$
\Sigma=\left[\begin{array}{ll}
-E\left(\frac{\delta^{2} \ln L}{\delta \theta^{2}}\right) & -E\left(\frac{\delta^{2} \ln L}{\delta \theta \delta \alpha}\right) \\
-E\left(\frac{\delta^{2} \ln L}{\delta \theta \delta \alpha}\right) & -E\left(\frac{\delta^{2} \ln L}{\delta \alpha^{2}}\right)
\end{array}\right]
$$

\section{Theorem}

The ML estimator $\underline{\hat{\theta}}$ of $\underline{\theta}=\left(\begin{array}{l}\theta \\ \alpha\end{array}\right)$ is consistent and asymptotically normal:

$$
\text { i.e. } \sqrt{n}(\underline{\hat{\theta}}-\underline{\theta}) \stackrel{d}{\rightarrow} N^{(2)}\left(\underline{0}, I^{-1}(\underline{\theta})\right)
$$

where

$$
I^{-1}(\underline{\theta})=N \Sigma^{-1}
$$

\section{Likelihood, Prior, Posterior and Marginal}

In this section we obtain the posterior and marginal posterior distributions of the parameters We write the likelihood function $\mathrm{L}$ define in $(2.1)$ as

$$
\begin{array}{r}
L=C \theta^{A_{1}-N}(1-\theta)^{A_{1}(\beta-1)+m N}(1-\alpha)^{n_{2}}(1-\theta \beta)^{N} \times\left[1+\alpha(m+2 \beta-1) \theta(1-\theta)^{\beta-1}\right]^{n_{1}} \\
=C \theta^{A_{1}-N}(1-\theta)^{A_{1}(\beta-1)+m N}(1-\alpha)^{n_{2}} \sum_{j=0}^{N}\left(\begin{array}{c}
N \\
j
\end{array}\right)(-\theta \beta)^{j} \times \\
\sum_{i=0}^{n_{1}}\left(\begin{array}{c}
n_{1} \\
i
\end{array}\right)\left[\alpha(m+2 \beta-1) \theta(1-\theta)^{\beta-1}\right]^{i} \\
=C \sum_{j=0}^{N} \sum_{i=0}^{n_{1}}\left(\begin{array}{c}
N \\
j
\end{array}\right)\left(\begin{array}{c}
n_{1} \\
i
\end{array}\right)(-\beta)^{j}(m+2 \beta-1)^{i} \theta^{A_{1}-N+j+i}(1-\theta)^{A_{1}(\beta-1)+m N+i(\beta-1)}(\alpha)^{i}(1-\alpha)^{n_{2}}
\end{array}
$$

where,

$$
C=(m+2 \beta-1)^{n_{2}} \prod_{i=3}^{k}\left[\frac{\Gamma(m+\beta i)}{(i-1) ! \Gamma(m+\beta i-i+1)}\right]^{n_{i}}
$$

Here, we assume beta priors of $\theta$ and $\alpha$ are independent and their prior distribution as beta distribution since they would be mathematically tractable with respect to the likelihood function.

The prior distribution of $\theta$ is :

$$
g_{1}(\theta)=\frac{1}{\beta\left(a_{1}, b_{1}\right)} \times \theta^{a_{1}-1}(1-\theta)^{b_{1}-1}, \quad 0<\theta<1
$$

and prior distribution of $\alpha$ is:

$$
g_{2}(\alpha)=\frac{1}{\beta\left(a_{2}, b_{2}\right)} \times \alpha^{a_{2}-1}(1-\alpha)^{b_{2}-1}, \quad 0<\alpha<1
$$

where $a_{1}, a_{2}, b_{1}, b_{2}>0$.

The mean and variance of the prior distribution of $\alpha$ are Mean $=\frac{a_{2}}{a_{2}+b_{2}} \quad$ and $\quad$ Variance $=\frac{a_{2 b_{2}}}{\left(a_{2}+b_{2}\right)^{2} \times\left(a_{2}+b_{2}+1\right)}$. Hence the joint prior distribution of $\theta$ and $\alpha$ will be

$$
g(\theta, \alpha)=\frac{1}{\beta\left(a_{1}, b_{1}\right) \beta\left(a_{2}, b_{2}\right)} \times \theta^{a_{1}-1}(1-\theta)^{b_{1}-1} \times \alpha^{a_{2}-1}(1-\alpha)^{b_{2}-1}
$$

and joint posterior density of $\theta$ and $\alpha$ can be obtained as

$$
\pi(\theta, \alpha \mid x)=\frac{L \times g(\theta, \alpha)}{m(x)}
$$

where $\mathrm{m}(\mathrm{x})$ is the marginal distribution of $\mathrm{x}$, given by

$$
\begin{aligned}
& m(x)=\int_{\theta} \int_{\alpha} L g(\theta, \alpha) d \theta d \alpha \\
= & \sum_{i} \sum_{j} c_{i j} \int_{\theta} \int_{\alpha} \theta^{A_{i j}+a_{1}-1} \times(1-\theta)^{B_{i}+b_{1}-1} \times \alpha^{i+a_{2}-1} \times(1-\alpha)^{n_{2}+b_{2}-1} d \theta d \alpha
\end{aligned}
$$




$$
\sum_{i} \sum_{j} c_{i j} \beta_{\left(A_{i j}+a_{1}, B_{i}+b_{1}\right)} \cdot \beta_{\left(i+a_{2}, n_{2}+b_{2}\right)},
$$

here

$$
A_{i j}=A_{1}-N+j+i, \quad B_{i}=\left(A_{1}+i\right)(\beta-1)+m N
$$

and

$$
c_{i j}=C \sum_{j=0}^{N} \sum_{i=0}^{n_{1}}\left(\begin{array}{c}
N \\
j
\end{array}\right)\left(\begin{array}{c}
n_{1} \\
i
\end{array}\right)(-\beta)^{j}(m+2 \beta-1)^{i} .
$$

Putting the value of equation (3.6) in equation (3.5), the joint posterior density of $\theta$ and $\alpha$ becomes

$$
\pi(\theta, \alpha \mid x)=\frac{\sum_{j=0}^{N} \sum_{i=0}^{n} c_{i j} \theta^{A_{i j}+a_{1}-1} \times(1-\theta)^{B_{i}+b_{1}-1} \times \alpha^{i+a_{2}-1} \times(1-\alpha)^{n_{2}+b_{2}-1}}{\sum_{i} \sum_{j} c_{i j} \beta\left(A_{i j}+a_{1}, B_{i}+b_{1}\right) \cdot \beta_{\left(i+a_{2}, n_{2}+b_{2}\right)}}
$$

The marginal posterior distribution of $\theta$ is given by

$$
\pi(\theta \mid x)=\frac{\sum_{j=0}^{N} \sum_{i=0}^{n_{1} c_{i j}} \theta^{A_{i j}+a_{1}-1} \times(1-\theta)^{B_{i}+b_{1}-1} \times \beta_{\left(i+a_{2}, n_{2}+b_{2}\right)}}{\sum_{i} \sum_{j} c_{i j} \beta_{\left(A_{i j}+a_{1}, B_{i}+b_{1}\right)^{-} \cdot \beta_{\left(i+a_{2}, n_{2}+b_{2}\right)}}}
$$

The marginal posterior distribution of $\alpha$ is given by

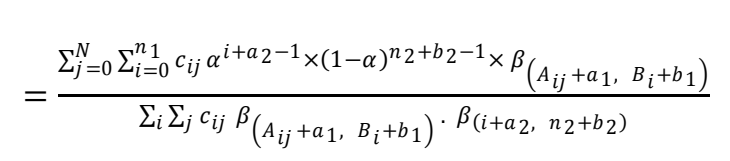$$
\pi(\alpha \mid x)=\int_{\theta} \pi(\theta, \alpha \mid x) d \theta
$$

where $0<\alpha<1$.

\section{Bayes estimation under general entropy loss function (GELF)}

The use of symmetric loss function is found to be inappropriate with respect to estimation procedure as an overestimation of reliability function is usually much more serious than an underestimation. In such situation, asymmetric loss function is more appropriate.

In Bayesian analysis, the loss function is used to represent a penalty associated with each of the possible estimators. As we know symmetric loss function assumes that positive and negative errors are equally serious. However, in some estimation problems such assumptions may be inappropriate. A positive error may be more serious than a negative error or vice - versa. For example, an overestimation of the reliability function is usually much more serious than an underestimation. In this situation, asymmetric loss functions are more appropriate. Here, we consider asymmetric loss function namely General Entropy loss function proposed by Calabria and Pulcini (1994) General Entropy Loss function has been proposed and adopted by some researchers in recent years. Now we derive the Bayes estimators of the parameter $\theta$ and $\alpha$.

The GELF is given by

$$
L_{1}(\widehat{\theta}, \theta)=\left(\frac{\widehat{\theta}}{\theta}\right)^{\alpha_{1}}-\alpha_{1} \ln \left(\frac{\widehat{\theta}}{\theta}\right)-1
$$

where $\alpha_{1} \neq 0, \quad 0<\theta<1$

The sign of the shape parameter $\alpha_{1}$ reflects the deviation of the asymmetry, $\alpha_{1}>0$. If overestimation is more serious than underestimation, and vice versa, and the magnitude of $\alpha_{1}$ reflects the degree of asymmetry. The Bayes estimator of $\theta$ under the GELF given in (4.1) is given by $\hat{\theta}_{G}$

To obtain Baye's estimator of $\theta$, let us consider

$$
\hat{\theta}_{G}=\left[E_{\pi}\left(\theta^{-\alpha_{1}}\right)\right]^{-1 / \alpha_{1}}
$$

$$
\begin{array}{r}
E_{\pi}\left(\theta^{-\alpha_{1}}\right)=\int_{\theta} \theta^{-\alpha_{1}} \pi(\theta / x) \cdot d \theta \\
E_{\pi}\left(\theta^{-\alpha_{1}}\right)=\int_{\theta} \theta^{-\alpha_{1}} \frac{\sum_{j=0}^{N} \sum_{i=0}^{n_{1}} c_{i j} \theta^{A_{i j}+a_{1}-1} \times(1-\theta)^{B_{i}+b_{1}-1} \times \beta_{\left(i+a_{2}, n_{2}+b_{2}\right)}}{\sum_{i} \sum_{j} c_{i j} \beta_{\left(A_{i j}+a_{1}, B_{i}+b_{1}\right)} \cdot \beta_{\left(i+a_{2}, n_{2}+b_{2}\right)}} d \theta \\
=\frac{\sum_{i} \sum_{j} c_{i j} \beta_{\left(A_{i j}+a_{1}-\alpha_{1}, B_{i}+b_{1}\right)} \beta_{\left(i+a_{2}, n_{2}+b_{2}\right)}}{\sum_{i} \sum_{j} c_{i j} \beta_{\left(A_{i j}+a_{1}, B_{i}+b_{1}\right)} \beta_{\left(i+a_{2}, n_{2}+b_{2}\right)}}
\end{array}
$$

Substituting in (4.2), we get the Bayes estimator of $\theta$ as 


$$
\hat{\theta}_{G}=\left[\frac{\sum_{i} \sum_{j} c_{i j} \beta_{\left(A_{i j}+a_{1}-\alpha_{1}, B_{i}+b_{1}\right)} \beta_{\left(i+a_{2}, n_{2}+b_{2}\right)}}{\sum_{i} \sum_{j} c_{i j} \beta_{\left(A_{i j}+a_{1}, B_{i}+b_{1}\right)} \beta_{\left(i+a_{2}, n_{2}+b_{2}\right)}}\right]^{-1 / \alpha_{1}}
$$

Similarly to obtain Bayes estimator of $\alpha$ we consider

$$
\begin{array}{r}
E_{\pi}\left(\alpha^{-\alpha_{1}}\right)=\int_{\alpha} \alpha^{-\alpha_{1}} \pi(\alpha / x) d \alpha \\
=\int_{\theta} \alpha^{-\alpha_{1}} \frac{\sum_{j=0}^{N} \sum_{i=0}^{n_{1}} c_{i j} \alpha^{i+a_{2}-1} \times(1-\alpha)^{n_{2}+b_{2}-1} \times \beta_{\left(A_{i j}+a_{1}, B_{i}+b_{1}\right)}}{\sum_{i} \sum_{j} c_{i j} \beta_{\left(A_{i j}+a_{1}, B_{i}+b_{1}\right)} \cdot \beta_{\left(i+a_{2}, n_{2}+b_{2}\right)}} d x \\
=\frac{\sum_{i} \sum_{j} c_{i j} \beta_{\left(i+a_{2}-\alpha_{1}, n_{2}+b_{2}\right)} \beta_{\left(A_{i j}+a_{1}, B_{i}+b_{1}\right)}}{\sum_{i} \sum_{j} c_{i j} \beta_{\left(A_{i j}+a_{1}, B_{i}+b_{1}\right)} \beta_{\left(i+a_{2}, n_{2}+b_{2}\right)}}
\end{array}
$$

According to (4.2), the Baye's estimator of $\alpha$ becomes

$$
\hat{\alpha}_{G}=\left[\frac{\sum_{i} \sum_{j} c_{i j} \beta_{\left(i+a_{2}-\alpha_{1}, n_{2}+b_{2}\right)} \beta_{\left(A_{i j}+a_{1}, B_{i}+b_{1}\right)}}{\sum_{i} \sum_{j} c_{i j} \beta_{\left(A_{i j}+a_{1}, B_{i}+b_{1}\right)} \beta_{\left(i+a_{2}, n_{2}+b_{2}\right)}}\right]^{-1 / \alpha_{1}}
$$

The Bayes estimates of $\theta$ and $\alpha$ under Squared Error Loss Function can be obtained by substituting $\alpha_{1}=-1$ in the above results (4.2) and (4.3).

\section{Simulation Study}

In this section we have generated 1000 different random samples each of size 25 by using Monte Carlo simulation with $\beta=2, m=1, \theta=0.05, \alpha=0.5$ from the MSBGNBD defined in equation (1.6) and obtained the ML estimates and Bayes estimates (under GELF) along with their simulated risk (SR) for different choice of the hyper parameters $a_{1}, a_{2}, b_{1}$, $b_{2}, \alpha_{1}$. The simulated results are shown in the following tables.

Table 1. ML estimates and Simulated risks of the parameters

\begin{tabular}{cccc}
\multicolumn{2}{c}{ MLE } & \multicolumn{2}{c}{ SR of } \\
$\ddot{\theta}$ & $\hat{\alpha}$ & 0 & $\hat{\theta}$ \\
0.056 & 0.451 & 0.020 & 0.318 \\
596 & 310 & 051 & 690
\end{tabular}


Table 2. $a_{2}, \quad b_{1}, \quad b_{2}$ are fixed and increase the value of $a_{1}$.

\begin{tabular}{|c|c|c|c|c|c|c|c|c|}
\hline$a_{1}$ & $a_{2}$ & $b_{1}$ & $b_{2}$ & $\alpha_{1}$ & $\hat{\theta}_{G}$ & $\hat{\alpha}_{G}$ & $\operatorname{SR}\left(\hat{\theta}_{G}\right)$ & $\operatorname{SR}\left(\hat{\alpha}_{G}\right)$ \\
\hline \multirow[t]{12}{*}{3} & 3 & 5 & 5 & 2 & 0.0395 & 0.2511 & 0.0154 & 0.2512 \\
\hline & & & & 3 & 0.0336 & 0.2146 & 0.0198 & 0.2871 \\
\hline & & & & 4 & 0.0296 & 0.1990 & 0.0225 & 0.3027 \\
\hline & & & & 5 & 0.0265 & 0.1974 & 0.0246 & 0.3046 \\
\hline & & & & 10 & 0.0217 & 0.2658 & 0.0283 & 0.2426 \\
\hline & & & & 20 & 0.0214 & 0.4765 & 0.0285 & 0.0808 \\
\hline & & & & -2 & 0.0604 & 0.4290 & 0.0137 & 0.0846 \\
\hline & & & & -3 & 0.0655 & 0.4506 & 0.0177 & 0.0680 \\
\hline & & & & -4 & 0.0697 & 0.4732 & 0.0213 & 0.0539 \\
\hline & & & & -5 & 0.0737 & 0.4925 & 0.0251 & 0.0480 \\
\hline & & & & -10 & 0.0919 & 0.5662 & 0.0424 & 0.0793 \\
\hline & & & & -20 & 0.1218 & 0.6517 & 0.0720 & 0.1572 \\
\hline \multirow[t]{12}{*}{5} & 3 & 5 & 5 & 2 & 0.0603 & 0.2878 & 0.0136 & 0.2174 \\
\hline & & & & 3 & 0.0551 & 0.2393 & 0.0108 & 0.2639 \\
\hline & & & & 4 & 0.0490 & 0.2167 & 0.0109 & 0.2860 \\
\hline & & & & 5 & 0.0425 & 0.2101 & 0.0134 & 0.2926 \\
\hline & & & & 10 & 0.0277 & 0.2588 & 0.0226 & 0.2491 \\
\hline & & & & 20 & 0.0237 & 0.4570 & 0.0263 & 0.0870 \\
\hline & & & & -2 & 0.0791 & 0.4602 & 0.0299 & 0.0668 \\
\hline & & & & -3 & 0.0835 & 0.4813 & 0.0342 & 0.0561 \\
\hline & & & & -4 & 0.0874 & 0.5025 & 0.0379 & 0.0513 \\
\hline & & & & -5 & 0.0914 & 0.5189 & 0.0418 & 0.0547 \\
\hline & & & & -10 & 0.1091 & 0.5828 & 0.0593 & 0.0974 \\
\hline & & & & -20 & 0.1377 & 0.6597 & 0.0878 & 0.1662 \\
\hline \multirow[t]{6}{*}{7} & 3 & 5 & 5 & 2 & 0.0798 & 0.3164 & 0.0306 & 0.1929 \\
\hline & & & & 3 & 0.0744 & 0.2660 & 0.0255 & 0.2393 \\
\hline & & & & 4 & 0.0694 & 0.2350 & 0.0211 & 0.2690 \\
\hline & & & & 5 & 0.0641 & 0.2198 & 0.0166 & 0.2833 \\
\hline & & & & 10 & 0.0372 & 0.2565 & 0.0143 & 0.2502 \\
\hline & & & & 20 & 0.0268 & 0.4518 & 0.0231 & 0.0866 \\
\hline & & & & -2 & 0.0968 & 0.4786 & 0.0471 & 0.0634 \\
\hline & & & & -3 & 0.1005 & 0.5010 & 0.0508 & 0.0573 \\
\hline & & & & -4 & 0.1045 & 0.5171 & 0.0547 & 0.0607 \\
\hline & & & & -5 & 0.1076 & 0.5382 & 0.0578 & 0.0675 \\
\hline & & & & -10 & 0.1238 & 0.6016 & 0.0739 & 0.1145 \\
\hline & & & & -20 & 0.1509 & 0.6735 & 0.1009 & 0.1795 \\
\hline
\end{tabular}


Table 3. $a_{1}, b_{1}, \quad b_{2}$ are fixed and increase the value of $a_{2}$

\begin{tabular}{|c|c|c|c|c|c|c|c|c|}
\hline$a_{1}$ & $a_{2}$ & $b_{1}$ & $b_{2}$ & $\alpha_{1}$ & $\hat{\theta}_{G}$ & $\hat{\alpha}_{G}$ & $\operatorname{SR}\left(\hat{\theta}_{G}\right)$ & $\operatorname{SR}\left(\hat{\alpha}_{G}\right)$ \\
\hline 3 & 3 & 5 & 5 & 2 & 0.0395 & 0.2511 & 0.0154 & 0.2512 \\
\hline & & & & 3 & 0.0336 & 0.2146 & 0.0198 & 0.2871 \\
\hline & & & & 4 & 0.0296 & 0.1990 & 0.0225 & 0.3027 \\
\hline & & & & 5 & 0.0265 & 0.1974 & 0.0246 & 0.3046 \\
\hline & & & & 10 & 0.0217 & 0.2658 & 0.0283 & 0.2426 \\
\hline & & & & 20 & 0.0214 & 0.4765 & 0.0285 & 0.0808 \\
\hline & & & & -2 & 0.0604 & 0.4290 & 0.0137 & 0.0846 \\
\hline & & & & -3 & 0.0655 & 0.4506 & 0.0177 & 0.0680 \\
\hline & & & & -4 & 0.0697 & 0.4732 & 0.0213 & 0.0539 \\
\hline & & & & -5 & 0.0737 & 0.4925 & 0.0251 & 0.0480 \\
\hline & & & & -10 & 0.0919 & 0.5662 & 0.0424 & 0.0793 \\
\hline & & & & -20 & 0.1218 & 0.6517 & 0.0720 & 0.1572 \\
\hline 3 & 5 & 5 & 5 & 2 & 0.0430 & 0.4267 & 0.0141 & 0.0878 \\
\hline & & & & 3 & 0.0364 & 0.3876 & 0.0179 & 0.1206 \\
\hline & & & & 4 & 0.0313 & 0.3294 & 0.0213 & 0.1756 \\
\hline & & & & 5 & 0.0280 & 0.2833 & 0.0234 & 0.2200 \\
\hline & & & & 10 & 0.0223 & 0.2586 & 0.0277 & 0.2470 \\
\hline & & & & 20 & 0.0217 & 0.4371 & 0.0282 & 0.0999 \\
\hline & & & & -2 & 0.0639 & 0.5291 & 0.0165 & 0.0556 \\
\hline & & & & -3 & 0.0691 & 0.5425 & 0.0208 & 0.0626 \\
\hline & & & & -4 & 0.0733 & 0.5580 & 0.0246 & 0.0735 \\
\hline & & & & -5 & 0.0778 & 0.5696 & 0.0288 & 0.0832 \\
\hline & & & & -10 & 0.0959 & 0.6237 & 0.0464 & 0.1315 \\
\hline & & & & -20 & 0.1253 & 0.6927 & 0.0755 & 0.1966 \\
\hline 3 & 7 & 5 & 5 & 2 & 0.0446 & 0.5275 & 0.0128 & 0.0536 \\
\hline & & & & 3 & 0.0383 & 0.5039 & 0.0166 & 0.0472 \\
\hline & & & & 4 & 0.0332 & 0.4741 & 0.0198 & 0.0528 \\
\hline & & & & 5 & 0.0292 & 0.4378 & 0.0224 & 0.0765 \\
\hline & & & & 10 & 0.0229 & 0.2825 & 0.0271 & 0.2218 \\
\hline & & & & 20 & 0.0218 & 0.4094 & 0.0281 & 0.1180 \\
\hline & & & & -2 & 0.0667 & 0.5931 & 0.0188 & 0.1034 \\
\hline & & & & -3 & 0.0713 & 0.6053 & 0.0228 & 0.1142 \\
\hline & & & & -4 & 0.0756 & 0.6162 & 0.0269 & 0.1243 \\
\hline & & & & -5 & 0.0803 & 0.6234 & 0.0313 & 0.1311 \\
\hline & & & & -10 & 0.0983 & 0.6667 & 0.0487 & 0.1717 \\
\hline & & & & -20 & 0.1276 & 0.7219 & 0.0778 & 0.2249 \\
\hline
\end{tabular}


Table 4. $a_{1}, a_{2}, b_{2}$ are fixed and increase the value of $b_{1}$

\begin{tabular}{|c|c|c|c|c|c|c|c|c|}
\hline$a_{1}$ & $a_{2}$ & $b_{1}$ & $b_{2}$ & $\alpha_{1}$ & $\hat{\theta}_{G}$ & $\hat{\alpha}_{G}$ & $\operatorname{SR}\left(\hat{\theta}_{G}\right)$ & $\operatorname{SR}\left(\hat{\alpha}_{G}\right)$ \\
\hline \multirow[t]{12}{*}{3} & 3 & 3 & 5 & 2 & 0.0408 & 0.2517 & 0.0148 & 0.2507 \\
\hline & & & & 3 & 0.0347 & 0.2153 & 0.0192 & 0.2866 \\
\hline & & & & 4 & 0.0296 & 0.2023 & 0.0224 & 0.2994 \\
\hline & & & & 5 & 0.0267 & 0.1996 & 0.0243 & 0.3023 \\
\hline & & & & 10 & 0.0224 & 0.2651 & 0.0276 & 0.2435 \\
\hline & & & & 20 & 0.0223 & 0.4694 & 0.0276 & 0.0846 \\
\hline & & & & -2 & 0.0621 & 0.4284 & 0.0151 & 0.0852 \\
\hline & & & & -3 & 0.0667 & 0.4535 & 0.0188 & 0.0664 \\
\hline & & & & -4 & 0.0706 & 0.4773 & 0.0221 & 0.0503 \\
\hline & & & & -5 & 0.0746 & 0.4974 & 0.0258 & 0.0463 \\
\hline & & & & -10 & 0.0935 & 0.5680 & 0.0440 & 0.0817 \\
\hline & & & & -20 & 0.1241 & 0.6505 & 0.0743 & 0.1559 \\
\hline \multirow[t]{12}{*}{3} & 3 & 5 & 5 & 2 & 0.0395 & 0.2511 & 0.0154 & 0.2512 \\
\hline & & & & 3 & 0.0336 & 0.2146 & 0.0198 & 0.2871 \\
\hline & & & & 4 & 0.0296 & 0.1990 & 0.0225 & 0.3027 \\
\hline & & & & 5 & 0.0265 & 0.1974 & 0.0246 & 0.3046 \\
\hline & & & & 10 & 0.0217 & 0.2658 & 0.0283 & 0.2426 \\
\hline & & & & 20 & 0.0214 & 0.4765 & 0.0285 & 0.0808 \\
\hline & & & & -2 & 0.0604 & 0.4290 & 0.0137 & 0.0846 \\
\hline & & & & -3 & 0.0655 & 0.4506 & 0.0177 & 0.0680 \\
\hline & & & & -4 & 0.0697 & 0.4732 & 0.0213 & 0.0539 \\
\hline & & & & -5 & 0.0737 & 0.4925 & 0.0251 & 0.0480 \\
\hline & & & & -10 & 0.0919 & 0.5662 & 0.0424 & 0.0793 \\
\hline & & & & -20 & 0.1218 & 0.6517 & 0.0720 & 0.1572 \\
\hline \multirow[t]{6}{*}{3} & 3 & 7 & 5 & 2 & 0.0388 & 0.2489 & 0.0159 & 0.2535 \\
\hline & & & & 3 & 0.0330 & 0.2135 & 0.0203 & 0.2883 \\
\hline & & & & 4 & 0.0291 & 0.1992 & 0.0232 & 0.3028 \\
\hline & & & & 5 & 0.0259 & 0.1962 & 0.0252 & 0.3058 \\
\hline & & & & 10 & 0.0210 & 0.2689 & 0.0290 & 0.2397 \\
\hline & & & & 20 & 0.0207 & 0.4778 & 0.0292 & 0.0811 \\
\hline & & & & -2 & 0.0589 & 0.4287 & 0.0123 & 0.0838 \\
\hline & & & & -3 & 0.0636 & 0.4524 & 0.0159 & 0.0655 \\
\hline & & & & -4 & 0.0683 & 0.4714 & 0.0202 & 0.0558 \\
\hline & & & & -5 & 0.0729 & 0.4878 & 0.0243 & 0.0496 \\
\hline & & & & -10 & 0.0906 & 0.5627 & 0.0412 & 0.0779 \\
\hline & & & & -20 & 0.1200 & 0.6502 & 0.0702 & 0.1556 \\
\hline
\end{tabular}


Table 5. $a_{1}, a_{2}, b_{1}$ are fixed and increase the value of $b_{2}$.

\begin{tabular}{|c|c|c|c|c|c|c|c|c|}
\hline$a_{1}$ & $a_{2}$ & $b_{1}$ & $b_{2}$ & $\alpha_{1}$ & $\hat{\theta}_{G}$ & $\hat{\alpha}_{G}$ & $\operatorname{SR}\left(\hat{\theta}_{G}\right)$ & $\operatorname{SR}\left(\hat{\alpha}_{G}\right)$ \\
\hline \multirow[t]{13}{*}{3} & 3 & 5 & 3 & 2 & 0.0424 & 0.3572 & 0.0134 & 0.1580 \\
\hline & & & & 3 & 0.0366 & 0.3058 & 0.0173 & 0.2040 \\
\hline & & & & 4 & 0.0314 & 0.2889 & 0.0209 & 0.2214 \\
\hline & & & & 5 & 0.0275 & 0.2921 & 0.0237 & 0.2196 \\
\hline & & & & 10 & 0.0223 & 0.3868 & 0.0277 & 0.1515 \\
\hline & & & & 20 & 0.0216 & 0.5980 & 0.0283 & 0.1339 \\
\hline & & & & & & & & \\
\hline & & & & -2 & 0.0650 & 0.5326 & 0.0170 & 0.0780 \\
\hline & & & & -3 & 0.0690 & 0.5608 & 0.0204 & 0.0910 \\
\hline & & & & -4 & 0.0733 & 0.5812 & 0.0244 & 0.1059 \\
\hline & & & & -5 & 0.0780 & 0.5937 & 0.0288 & 0.1148 \\
\hline & & & & -10 & 0.0966 & 0.6559 & 0.0470 & 0.1685 \\
\hline & & & & -20 & 0.1262 & 0.7293 & 0.0764 & 0.2351 \\
\hline & & & & & & & & \\
\hline \multirow[t]{13}{*}{3} & 3 & 5 & 5 & 2 & 0.0395 & 0.2511 & 0.0154 & 0.2512 \\
\hline & & & & 3 & 0.0336 & 0.2146 & 0.0198 & 0.2871 \\
\hline & & & & 4 & 0.0296 & 0.1990 & 0.0225 & 0.3027 \\
\hline & & & & 5 & 0.0265 & 0.1974 & 0.0246 & 0.3046 \\
\hline & & & & 10 & 0.0217 & 0.2658 & 0.0283 & 0.2426 \\
\hline & & & & 20 & 0.0214 & 0.4765 & 0.0285 & 0.0808 \\
\hline & & & & & & & & \\
\hline & & & & -2 & 0.0604 & 0.4290 & 0.0137 & 0.0846 \\
\hline & & & & -3 & 0.0655 & 0.4506 & 0.0177 & 0.0680 \\
\hline & & & & -4 & 0.0697 & 0.4732 & 0.0213 & 0.0539 \\
\hline & & & & -5 & 0.0737 & 0.4925 & 0.0251 & 0.0480 \\
\hline & & & & -10 & 0.0919 & 0.5662 & 0.0424 & 0.0793 \\
\hline & & & & -20 & 0.1218 & 0.6517 & 0.0720 & 0.1572 \\
\hline & & & & & & & & \\
\hline \multirow[t]{13}{*}{3} & 3 & 5 & 7 & 2 & 0.0371 & 0.1910 & 0.0176 & 0.3096 \\
\hline & & & & 3 & 0.0320 & 0.1625 & 0.0212 & 0.3380 \\
\hline & & & & 4 & 0.0278 & 0.1521 & 0.0240 & 0.3484 \\
\hline & & & & 5 & 0.0255 & 0.1490 & 0.0256 & 0.3516 \\
\hline & & & & 10 & 0.0213 & 0.1886 & 0.0287 & 0.3137 \\
\hline & & & & 20 & 0.0214 & 0.3710 & 0.0285 & 0.1454 \\
\hline & & & & & & & & \\
\hline & & & & -2 & 0.0576 & 0.3531 & 0.0120 & 0.1500 \\
\hline & & & & -3 & 0.0622 & 0.3770 & 0.0154 & 0.1274 \\
\hline & & & & -4 & 0.0664 & 0.3988 & 0.0187 & 0.1066 \\
\hline & & & & -5 & 0.0706 & 0.4176 & 0.0222 & 0.0886 \\
\hline & & & & -10 & 0.0889 & 0.4922 & 0.0395 & 0.0338 \\
\hline & & & & -20 & 0.1188 & 0.5846 & 0.0691 & 0.0912 \\
\hline
\end{tabular}

\section{Conclusions}

From the Table 1 and 5, we observe the following conclusions.

- For given $\alpha$, and smaller value of $a_{1}$, Bayes estimator under GELF is more precise than ML estimates.

- For small value of $a_{1}$ and $\alpha_{1}\left(a_{2}, b_{1}, b_{2}\right.$ are fixed), Bayes estimates under GELF is more precise than ML estimates.

- For small value of $a_{2}$ and $\alpha_{1}\left(a_{1}, b_{1}, b_{2}\right.$ are fixed), Bayes estimates under GELF is more precise than ML estimates.

- For small value of $b_{1}$ and $\alpha_{1}\left(a_{1}, a_{2}, b_{2}\right.$ are fixed), Bayes estimates under GELF is more precise than ML estimates.

- For small value of $b_{2}$ and $\alpha_{1}\left(a_{1}, a_{2}, b_{1}\right.$ are fixed), Bayes estimates under GELF is more precise than ML estimates.

We also observe the following conclusions for Bayes estimator:

- For fixed value of $a_{1}, a_{2}$ and $b_{2}$ as $b_{1}$ increases, simulated risk of the Bayes estimates under GELF increases.

- For fixed value of $a_{1}, a_{2}$ and $b_{1}$ as $b_{2}$ increases, simulated risk of the Bayes estimates under GELF increases. 


\section{REFERENCES}

[1] Calabria, R. and Pulcini, G. (1994): An engineering approach to bayes estimation for the Weibull distribution, Micro Electron Reliability, 34, $789-802$.

[2] Cohen, A. C. (1960): Estimating the parameters of a modified Poisson distribution, JASA, 55, 139 - 143.

[3] Greenwood, M and Yule, G. U. (1920): An enquiry in to the nature of frequency distribution of multiple happenings with particular reference to the occurrence of multiple attacks of disease or repeated accidents, JRSS, Series-A, 83, 255- 279.

[4] Gupta R. C. (1979): Waiting time paradox and size biased sampling. Commun. Stat., Theory Methods A $813,601-607$.

[5] Gupta R. C. (1984): Some characterizations of renewal densities with emphasis in reliability, Math. Operationsforsch. Stat. 15, 571 -579 .

[6] Gupta R. C., Tripathi R. C. (1987): A comparison between the ordinary and the length - biased modified power series distributions with applications, Commun. Stat., Theory Methods 16, $1195-1206$.

[7] Gupta R. C., Tripathi R. C. (1992): Statistical inference based on the length - biased data for the modified power series distributions. Commun. Stat., Theory Methods 21, $519-537$.

[8] Hassan A., and Ahmad P.B. (2009): Misclassified size - biased modified power series distribution and its applications, Mathematica Bohemica, 134, 1 - 17

[9] Jain G. C. and Consul P. C. (1971): A generalized negative binomial distribution, SIAM J. Appl. Math, 21, 501 - 513.

[10] Jani P. N. and Shah S. M. (1979): Misclassification in modified power series distribution in which the value one is sometimes reported as zero and some of its applications, Metron, XXXVII, N. $3-4,121-136$.

[11] Noack, A. (1950): A class of random variables with discrete distribution, Annals of mathematical statistics, 22,127 - 132 .

[12] Patel A. I., Patel I. D. (2001): Misclassification in modified power series distribution and its applications. Assam statistical Review $15,55-69$.

[13] Patel A. I., Patel I. D (1996): Recurrence relations for moments of the so called misclassified distribution. Assam Statistical Review $10,107-119$

[14] Williford W. O., Bingham S. F. (1979): Bayesian Estimation of the parameters in two modified Poisson distributions, Commun Stat., Theory Methods A $813,1315-1326$.

[15] Wise, M. E. (1946): Thev use of the negative binomial distribution in an industrial sampling problem, JRSS, Series-B, 8, $202-211$. 